Pacific Journal of Mathematics

ON THE MAXIMAL MONOTONICITY OF SUBDIFFERENTIAL 


\title{
ON THE MAXIMAL MONOTONICITY OF SUBDIFFERENTIAL MAPPINGS
}

\author{
R. T. ROCKAFELLAR
}

The subdifferential of a lower semicontinuous proper convex function on a Banach space is a maximal monotone operator, as well as a maximal cyclically monotone operator. This result was announced by the author in a previous paper, but the argument given there was incomplete; the result is proved here by a different method, which is simpler in the case of reflexive Banach spaces. At the same time, a new fact is established about the relationship between the subdifferential of a convex function and the subdifferential of its conjugate in the nonreflexive case.

Let $E$ be a real Banach space with dual $E^{*}$. A proper convex function on $E$ is a function $f$ from $E$ to $(-\infty,+\infty]$, not identically $+\infty$, such that

$$
f((1-\lambda) x+\lambda y) \leqq(1-\lambda) f(x)+\lambda f(y)
$$

whenever $x \in E, y \in E$ and $0<\lambda<1$. The subdifferential of such a function $f$ is the (generally multivalued) mapping $\partial f: E \rightarrow E^{*}$ defined by

$$
\partial f(x)=\left\{x^{*} \in E^{*} \mid f(y) \geqq f(x)+\left\langle y-x, x^{*}\right\rangle, \forall y \in E\right\},
$$

where $\langle\cdot, \cdot\rangle$ denotes the canonical pairing between $E$ and $E^{*}$.

A multivalued mapping $T: E \rightarrow E^{*}$ is said to be a monotone operator if

$$
\left\langle x_{0}-x_{1}, x_{0}^{*}-x_{1}^{*}\right\rangle \geqq 0 \quad \text { whenever } \quad x_{0}^{*} \in T\left(x_{0}\right), x_{1}^{*} \in T\left(x_{1}\right) .
$$

It is said to be a cyclically monotone operator if

$$
\begin{gathered}
\left\langle x_{0}-x_{1}, x_{0}^{*}\right\rangle+\cdots+\left\langle x_{n-1}-x_{n}, x_{n-1}^{*}\right\rangle+\left\langle x_{n}-x_{0}, x_{n}^{*}\right\rangle \geqq 0 \\
\text { whenever } x_{i}^{*} \in T\left(x_{i}\right), i=0, \cdots, n .
\end{gathered}
$$

It is called a maximal monotone operator (resp. maximal cyclically monotone operator) if, in addition, its graph

$$
G(T)=\left\{\left(x, x^{*}\right) \mid x^{*} \in T(x)\right\} \subset E \times E^{*}
$$

is not properly contained in the graph of any other monotone (resp. cyclically monotone) operator $T^{\prime}: E \rightarrow E^{*}$.

This note is concerned with proving the following theorems. 
THEOREM A. If $f$ is a lower semicontinuous proper convex function on $E$, then $\partial f$ is a maximal monotone operator from $E$ to $E^{*}$.

THEOREM B. Let $T: E \rightarrow E^{*}$ be a multivalued mapping. In order that there exist a lower semicontinuous proper convex function $f$ on $E$ such that $T=\partial f$, it is necessary and sufficient that $T$ be a maximal cyclically monotone operator. Moreover, in this case $T$ determines $f$ uniquely up to an additive constant.

These theorems have previously been stated by us in [4] as Theorem 4 and Theorem 3, respectively. However, a gap occurs in the proofs in [4], as has kindly been brought to our attention recently by H. Brézis. (It is not clear whether formula (4.7) in the proof of Theorem 3 of [4] will hold for $\varepsilon$ sufficiently small, because $x_{i}^{*}$ depends on $\varepsilon$ and could conceivably increase unboundedly in norm as $\varepsilon$ decreases to 0 . The same oversight appears in the penultimate sentence of the proof of Theorem 4 of [4]). In view of this oversight, the proofs in [4] are incomplete; further arguments must be given before the maximality in Theorem A, the maximality in the necessary condition in Theorem B, and the uniqueness in Theorem $\mathrm{B}$ can be regarded as established. Such arguments will be given here.

2. Preliminary result. Let $f$ be a lower semicontinuous proper convex function on $E$. (For proper convex functions, lower semicontinuity in the strong topology of $E$ is the same as lower semicontinuity in the weak topology.) The conjugate of $f$ is the function $f^{*}$ on $E^{*}$ defined by

$$
f^{*}\left(x^{*}\right)=\sup \left\{\left\langle x, x^{*}\right\rangle-f(x) \mid x \in E\right\} .
$$

It is known that $f^{*}$ is a weak ${ }^{*}$ lower semicontinuous (and hence strongly lower semicontinuous) proper convex function on $E^{*}$, and that

$$
\begin{gathered}
f(x)+f^{*}\left(x^{*}\right)-\left\langle x, x^{*}\right\rangle \geqq 0, \forall x \in E, \forall x^{*} \in E^{*}, \\
\text { with equality if and only if } x^{*} \in \partial f(x)
\end{gathered}
$$

(see Moreau [3, §6]). The subdifferential $\partial f^{*}$, which is a multivalued mapping from $E^{*}$ to the bidual $E^{* *}$, can be compared with the subdifferential $\partial f$ from $E$ to $E^{*}$, when $E$ is regarded in the canonical way as a weak ${ }^{* *}$ dense subspace of $E^{* *}$ (the weak** topology being the weak topology induced on $E^{* *}$ by $E^{*}$ ). Facts about the relationship between $\partial f^{*}$ and $\partial f$ will be used below in proving Theorems $\mathrm{A}$ and $\mathrm{B}$.

In terms of the conjugate $f^{* *}$ of $f^{*}$, which is the weak ${ }^{* *}$ lower semicontinuous proper convex function on $E^{* *}$ defined by 


$$
f^{* *}\left(x^{* *}\right)=\sup \left\{\left\langle x^{* *}, x^{*}\right\rangle-f^{*}\left(x^{*}\right) \mid x^{*} \in E^{*}\right\},
$$

we have, as in (2.2),

$$
\begin{gathered}
f^{* *}\left(x^{* *}\right)+f^{*}\left(x^{*}\right)-\left\langle x^{* *}, x^{*}\right\rangle \geqq 0, \forall x^{* *} \in E^{* *}, \forall x^{*} \in E^{*}, \\
\text { with equality if and only if } x^{* *} \in \partial f^{*}\left(x^{*}\right) .
\end{gathered}
$$

Moreover, the restriction of $f^{* *}$ to $E$ is $f$ (see $[3, \S 6]$ ). Thus, if $E$ is reflexive, we can identify $f^{* *}$ with $f$, and it follows from (2.2) and (2.4) that $\partial f^{*}$ is just the "inverse" of $\partial f$, in other words one has $x \in \partial f^{*}\left(x^{*}\right)$ if and only if $x^{*} \in \partial f(x)$. If $E$ is not reflexive, the relationship between $\partial f^{*}$ and $\partial f$ is more complicated, but $\partial f^{*}$ and $\partial f$ still completely determine each other, according to the following result.

Proposition 1. Let $f$ be a lower semicontinuous proper convex function on $E$, and let $x^{*} \in E^{*}$ and $x^{* *} \in E^{* *}$. Then $x^{* *} \in \partial f^{*}\left(x^{*}\right)$ if and only if there exists a net $\left\{x_{i}^{*} \mid i \in I\right\}$ in $E^{*}$ converging to $x^{*}$ in the strong topology and a bounded net $\left\{x_{i} \mid i \in I\right\}$ in $E$ (with the same partially ordered index set I) converging to $x^{* *}$ in the weak** topology, such that $x_{i}^{*} \in \partial f\left(x_{i}\right)$ for every $i \in I$.

Proof. The sufficiency of the condition is easy to prove. Given nets as described, we have

$$
f\left(x_{i}\right)+f^{*}\left(x_{i}^{*}\right)=\left\langle x_{i}, x_{i}^{*}\right\rangle, \forall i \in I
$$

by (2.2), where $f\left(x_{i}\right)=f^{* *}\left(x_{i}\right)$. Then by the lower semicontinuity of $f^{*}$ and $f^{* *}$ we have

$$
\begin{aligned}
f^{* *}\left(x^{* *}\right)+f^{*}\left(x^{*}\right) & \leqq \liminf \left\{f^{* *}\left(x_{i}\right)+f^{*}\left(x_{i}^{*}\right)\right\} \\
& =\lim \left\langle x_{i}, x_{i}^{*}\right\rangle=\left\langle x^{* *}, x^{*}\right\rangle .
\end{aligned}
$$

(The last equality makes use of the boundedness of the norms $\left\|x_{i}\right\|$, $i \in I$.) Thus $x^{* *} \in \partial f^{*}\left(x^{*}\right)$ by (2.4).

To prove the necessity of the condition, we demonstrate first that, given any $x^{* *} \in E^{* *}$, there exists a bounded net $\left\{y_{i} \mid i \in I\right\}$ in $E$ such that $y_{i}$ converges to $x^{* *}$ in the weak ${ }^{* *}$ topology and

$$
\lim f\left(y_{i}\right)=f^{* *}\left(x^{* *}\right) .
$$

Consider $f+h_{\alpha}$, where $\alpha$ is a positive real number and $h_{\alpha}$ is the lower semicontinuous proper convex function on $E$ defined by

$$
h_{\alpha}(x)=0 \text { if }\|x\| \leqq \alpha, h_{\alpha}(x)=+\infty \quad \text { if }\|x\|>\alpha .
$$

Assuming that $\alpha$ is sufficiently large, there exist points $x$ at which $f$ and $h_{\alpha}$ are both finite and $h_{\alpha}$ is continuous (i.e., points $x$ such that $f(x)<+\infty$ and $\|x\|<\alpha)$. Then, by the formulas for conjugates of 
sums of convex functions (see Moreau [3, pp. 38, 56, 57] or Rockafellar [5, Th. 3]), we have $\left(f+h_{\alpha}\right)^{*}=f^{*} \square h_{\alpha}^{*}$ (infimal convolution), and consequently

$$
\left(f+h_{\alpha}\right)^{* *}=\left(f^{*} \square h_{\alpha}^{*}\right)^{*}=f^{* *}+h_{\alpha}^{* *} .
$$

Moreover $h_{\alpha}^{*}\left(x^{*}\right)=\alpha\left\|x^{*}\right\|$ for ever $x^{*} \in E^{*}$, so that

$$
\begin{aligned}
h_{\alpha}^{* *}\left(x^{* *}\right) & =\sup \left\{\left\langle x^{* *}, x^{*}\right\rangle-\alpha\left\|x^{*}\right\| \mid x^{*} \in E^{*}\right\} \\
& = \begin{cases}0 & \text { if }\left\|x^{* *}\right\| \leqq \alpha, \\
+\infty & \text { if }\left\|x^{* *}\right\|>\alpha .\end{cases}
\end{aligned}
$$

Hence by (2.7), given any $x^{* *} \in E^{* *}$, we have

$$
f^{* *}\left(x^{* *}\right)=\left(f+h_{\alpha}\right)^{* *}\left(x^{* *}\right)
$$

for sufficiently large $\alpha>0$. On the other hand, it is known that, for any lower semicontinuous proper convex function $g$ on $E, g^{* *}$ is the greatest weak ${ }^{* *}$ lower semicontinuous function on $E^{* *}$ majorized by $g$ on $E$ (see $[3, \S 6]$ ), so that

$$
g^{* *}\left(x^{* *}\right)=\liminf _{y \rightarrow x^{* *}} g(y),
$$

where the "lim inf" is taken over all nets in $E$ converging to $x^{* *}$ in the weak** topology. Taking $g=f+h_{\alpha}$, we see from (2.8) and (2.9) that

$$
f^{* *}\left(x^{* *}\right)=\liminf _{y \rightarrow x^{*}}\left[f(y)+h_{\alpha}(y)\right],
$$

implying that (2.5) holds as desired for some net $\left\{y_{i} \mid i \in I\right\}$ in $E$ such that $y_{i}$ converges to $x^{* *}$ in the weak $^{* *}$ topology and $\left\|y_{i}\right\| \leqq \alpha$ for every $i \in I$.

Now, given any $x^{*} \in E^{*}$ and $x^{* *} \in \partial f^{*}\left(x^{*}\right)$, let $\left\{y_{i} \mid i \in I\right\}$ be a bounded net in $E$ such that $y_{i}$ converges to $x^{* *}$ in the weak** topology and (2.5) holds. Define $\varepsilon_{i} \geqq 0$ by

$$
\varepsilon_{i}^{2}=f\left(y_{i}\right)+f^{*}\left(x^{*}\right)-\left\langle y_{i}, x^{*}\right\rangle .
$$

Note that $\lim \varepsilon_{i}=0$ by (2.5) and (2.4). According to a lemma of Brondsted and Rockafellar [1, p. 608], there exist for each $i \in I$ an $x_{i} \in E$ and an $x_{i}^{*} \in E^{*}$ such that

$$
x_{i}^{*} \in \partial f\left(x_{i}\right),\left\|x_{i}-y_{i}\right\| \leqq \varepsilon_{i},\left\|x_{i}^{*}-x^{*}\right\| \leqq \varepsilon_{i} .
$$

The latter two conditions imply that the net $\left\{x_{i}^{*} \mid i \in I\right\}$ converges to $x^{*}$ in the strong topology of $E^{*}$, while the net $\left\{x_{i} \mid i \in I\right\}$ is bounded and converges to $x^{* *}$ in the weak ${ }^{* *}$ topology of $E^{* *}$. This completes the proof of Proposition 1. 
3. Proofs of Theorems $A$ and $B$. In the sequel, $f$ denotes a lower semicontinuous proper convex function on $E$, and $j$ denotes the continuous convex function $E$ defined by $j(x)=(1 / 2)\|x\|^{2}$. We shall make use of the fact that, for each $x \in E, \partial f(x)$ is by definition a certain (possibly empty, possibly unbounded) weak* closed convex subset of $E^{*}$, whereas $\partial j(x)$ is (by the finiteness and continuity of $j$, see $\left[3\right.$, p. 60]) a certain nonempty weak* compact convex subset of $E^{*}$. Furthermore

$$
\partial(f+j)=\partial f(x)+\partial j(x), \forall x \in E
$$

(see $\left[3\right.$, p. 62] or $\left[5\right.$, Th. 3]). The conjugate of $j$ is given by $j^{*}\left(x^{*}\right)=$ $(1 / 2)\left\|x^{*}\right\|^{2}$, and since

$$
(f+j)^{*}\left(x^{*}\right)=\left(f^{*} \square j^{*}\right)\left(x^{*}\right)=\min _{y^{*} \in E^{*}}\left\{f^{*}\left(y^{*}\right)+j^{*}\left(x^{*}-y^{*}\right)\right\}
$$

([3, §9] or [5, Th. 3]) the conjugate function $(f+j)^{*}$ is finite and continuous throughout $E^{*}$.

Proof of Theorem A. Theorem A has already been established by Minty [2] in the case of convex functions which, like $j$, are everywhere finite and continuous. Applying Minty's result to the function $(f+j)^{*}$, we may conclude that $\partial(f+j)^{*}$ is a maximal monotone operator from $E^{*}$ to $E^{* *}$. We shall show this implies that $\partial f$ is a maximal monotone operator from $E$ to $E^{*}$.

Let $T$ be a monotone operator from $E$ to $E^{*}$ such that the graph of $T$ includes the graph of $\partial f$, i.e.,

$$
T(x) \supset \partial f(x), \forall x \in E .
$$

We must show that equality necessarily holds in (3.2).

The mapping $T+\partial j$ defined by

$$
\begin{aligned}
(T+\partial j)(x) & =T(x)+\partial j(x) \\
& =\left\{x_{1}^{*}+x_{2}^{*} \mid x_{1}^{*} \in T(x), x_{2}^{*} \in \partial j(x)\right\}
\end{aligned}
$$

is a monotone operator from $E$ to $E^{*}$, since $T$ and $\partial j$ are, and by (3.1) and (3.2) we have

$$
(T+\partial j)(x) \supset \partial(f+j)(x), \forall x \in E .
$$

Let $S$ be the multivalued mapping from $E^{*}$ to $E^{* *}$ defined as follows: $x^{* *} \in S\left(x^{*}\right)$ if and only if there exists a net $\left\{x_{i}^{*} \mid i \in I\right\}$ in $E^{*}$ converging to $x^{*}$ in the strong topology, and a bounded net $\left\{x_{i} \mid i \in I\right\}$ in $E$ (with the same partially ordered index set $I$ ) converging to $x^{* *}$ in the weak ${ }^{* *}$ topology, such that

$$
x_{i}^{*} \in(T+\partial j)\left(x_{i}\right), \forall i \in I .
$$


It is readily verified that $S$ is a monotone operator. (The boundedness of the nets $\left\{x_{i} \mid i \in I\right\}$ enters in here.) Moreover

$$
S\left(x^{*}\right) \supset \partial(f+j)^{*}\left(x^{*}\right), \forall x^{*} \in E^{*},
$$

by (3.3) and Proposition 1. Since $\partial(f+j)^{*}$ is a maximal monotone operator, equality must actually hold in (3.4). This shows that one has $x \in \partial(f+j)^{*}\left(x^{*}\right)$ whenever $x \in E$ and $x \in S\left(x^{*}\right)$, hence in particular whenever $x^{*} \in(T+\partial j)(x)$. On the other hand, one always has $x^{*} \in \partial(f+j)(x)$ if $x \in \partial(f+j)^{*}\left(x^{*}\right)$ and $x \in E$. (This follows from applying (2.2) and (2.4) to $f+j$ in place of $f$.) Thus one has $x^{*} \in \partial(f+j)(x)$ if $x^{*} \in(T+\partial j)(x)$, implying by (3.3) and (3.1) that

$$
T(x)+\partial j(x)=\partial f(x)+\partial j(x), \forall x \in E .
$$

We shall show now from (3.5) that actually

$$
T(x)=\partial f(x), \forall x \in E,
$$

so that $\partial f$ must be a maximal monotone operator as claimed. Suppose that $x \in E$ is such that the inclusion in (3.2) is proper. This will lead to a contradiction. Since $\partial f(x)$ is a weak* closed convex subset of $E^{*}$, there must exist some point of $T(x)$ which can be separated strictly from $\partial f(x)$ be a weak* closed hyperplane. Thus, for a certain $y \in E$, we have

$$
\sup \left\{\left\langle y, x^{*}\right\rangle \mid x^{*} \in T(x)\right\}>\sup \left\{\left\langle y, x^{*}\right\rangle \mid x^{*} \in \partial f(x)\right\} .
$$

But then

$$
\begin{aligned}
& \sup \left\{\left\langle y, z^{*}\right\rangle \mid z^{*} \in T(x)+\partial j(x)\right\} \\
= & \sup \left\{\left\langle y, x^{*}\right\rangle \mid x^{*} \in T(x)\right\}+\sup \left\{\left\langle y, y^{*}\right\rangle \mid y^{*} \in \hat{o} j(x)\right\} \\
> & \sup \left\{\left\langle y, x^{*}\right\rangle \mid x^{*} \in \partial f(x)\right\}+\sup \left\{\left\langle y, y^{*}\right\rangle \mid y^{*} \in \hat{o} j(x)\right\} \\
= & \sup \left\{\left\langle y, z^{*}\right\rangle \mid z^{*} \in \partial f(x)+\partial j(x)\right\},
\end{aligned}
$$

inasmuch as $\partial j(x)$ is a nonempty bounded set, and this inequality is incompatible with (3.5).

Proof of Theorem B. Let $g$ be a lower semicontinuous proper convex function on $E$ such that

$$
\partial g(x) \supset \partial f(x), \forall x \in E .
$$

As noted at the beginning of the proof Theorem 3 of [4], to prove Theorem B it suffices, in view of Theorem 1 of [4] and its Corollary 2 , to demonstrate that $g=f+$ const.

We consider first the case where $f$ and $g$ are everywhere finite and continuous. Then, for each $x \in E, \partial f(x)$ is a nonempty weak* 
compact set, and

$$
f^{\prime}(x ; u)=\max \left\{\left\langle u, x^{*}\right\rangle \mid x^{*} \in \partial f(x)\right\}, \forall u \in E,
$$

where

$$
f^{\prime}(x ; u)=\lim _{\lambda \downarrow 0}[f(x+\lambda u)-f(x)] / \lambda
$$

[3, p. 65]. Similarly, $\partial g(x)$ is a nonempty weak* compact set, and

$$
g^{\prime}(x ; u)=\max \left\{\left\langle u, x^{*}\right\rangle \mid x^{*} \in \partial g(x)\right\}, \forall u \in E .
$$

It follows from (3.6), (3.7) and (3.8) that

$$
f^{\prime}(x ; u) \leqq g^{\prime}(x ; u), \forall x \in E, \forall u \in E .
$$

On the other hand, for any $x \in E$ and $y \in E$, we have

$$
\begin{aligned}
& f(y)-f(x)=\int_{0}^{1} f^{\prime}((1-\lambda) x+\lambda y ; y-x) d \lambda, \\
& g(y)-g(x)=\int_{0}^{1} g^{\prime}((1-\lambda) x+\lambda y ; y-x) d \lambda
\end{aligned}
$$

(see $[6, \S 24])$, so that by $(3.9)$ we have

$$
f(y)-f(x) \leqq g(y)-g(x), \forall x \in E, \forall y \in E .
$$

Of course, the latter can hold only if $g=f+$ const.

In the general case, we observe from (3.6) that

$$
\partial g(x)+\partial j(x) \supset \partial f(x)+\partial j(x), \forall x \in E,
$$

and consequently

$$
\partial(g+j)(x) \supset \partial(f+j)(x), \forall x \in E,
$$

by (3.1)(and its counterpart for $g$ ). This implies by Proposition 1 that

$$
\partial(g+j)^{*}\left(x^{*}\right) \supset \partial(f+j)^{*}\left(x^{*}\right), \forall x^{*} \in E^{*} .
$$

The functions $(f+j)^{*}$ and $(g+j)^{*}$ are finite and continuous on $E^{*}$, so we may conclude from (3.10) and the case already considered that

$$
(g+j)^{*}=(f+j)^{*}+\alpha
$$

for a certain real constant $\alpha$. Taking conjugates, we then have

$$
(g+j)^{* *}=(f+j)^{* *}-\alpha .
$$

Since $(g+j)^{* *}$ and $(f+j)^{* *}$ agree on $E$ with $g+j$ and $f+j$, respectively, (3.11) implies that

$$
g+j=f+j-\alpha
$$


and hence that $g=f+$ const.

REMARK. The preceding proofs become much simpler if $E$ is reflexive, since then $\partial f^{*}$ and $\partial(f+j)^{*}$ are just the "inverses" of $\partial f$ and $\partial(f+j)$, respectively, and Proposition 1 is superfluous. In this case, $S$ may be replaced by the inverse of $T+\partial j$ in the proof of Theorem A.

\section{REFERENCES}

1. A. Brondsted and R. T. Rockafellar, On the subdifferentiability of convex functions, Proc. Amer. Math. Soc. 16 (1965), 605-611.

2. G. J. Minty, On the monotonicity of the gradient of a convex function, Pacific J. Math. 14 (1964), 243-247.

3. J.-J. Moreau, Fonctionelles convexes, mimeographed lecture notes, Collège de France, 1967.

4. R. T. Rockafeller, Characterization of the subdifferentials of convex functions, Pacific J. Math. 17 (1966), 497-510.

5. ——, An extension of Fenchel's duality theorem, Duke Math. J. 33 (1966), 8190.

6. Convex analysis, Princeton University Press, 1969.

Received July 17, 1969. This work was supported in part by the Air Force Office of Scientific Research under grant AF-AFOSR-1202-67.

The University of Washington, Seattle 


\section{PACIFIC JOURNAL OF MATHEMATICS}

\section{EDITORS}

H. SAMELSON

Stanford University

Stanford, California 94305

\section{RichaRd PIERCe}

University of Washington

Seattle, Washington 98105
J. DUGUNDJI

Department of Mathematics

University of Southern California

Los Angeles, California 90007

RICHARD ARENS

University of California

Los Angeles, California 90024

\section{ASSOCIATE EDITORS}

E. F. BECKENBACH

B. H. NeUManN

F. WOLF

K. YosHIDA

\section{SUPPORTING INSTITUTIONS}

UNIVERSITY OF BRITISH COLUMBIA CALIFORNIA INSTITUTE OF TECHNOLOGY UNIVERSITY OF CALIFORNIA MONTANA STATE UNIVERSITY

UNIVERSITY OF NEVADA

NEW MEXICO STATE UNIVERSITY

OREGON STATE UNIVERSITY

UNIVERSITY OF OREGON

OSAKA UNIVERSITY

UNIVERSITY OF SOUTHERN CALIFORNIA
STANFORD UNIVERSITY

UNIVERSITY OF TOKYO

UNIVERSITY OF UTAH

WASHINGTON STATE UNIVERSITY

UNIVERSITY OF WASHINGTON

${ }^{*} \quad{ }^{*} \quad{ }^{*}$
AMERICAN MATHEMATICAL SOCIETY
CHEVRON RESEARCH CORPORATION
TRW SYSTEMS
NAVAL WEAPONS CENTER




\section{Pacific Journal of Mathematics}

\section{Vol. 33, No. $1 \quad$ March, 1970}

Mir Maswood Ali, On some extremal simplexes ................... 1

Silvio Aurora, On normed rings with monotone multiplication........... 15

Silvio Aurora, Normed fields which extend normed rings of integers....... 21

John Kelly Beem, Indefinite Minkowski spaces..................... 29

T. F. Bridgland, Trajectory integrals of set valued functions ........... 43

Robert Jay Buck, A generalized Hausdorff dimension for functions and sets ......................................... 69

Vlastimil B. Dlab, A characterization of perfect rings . . . . . . . . . . . . 79

Edward Richard Fadell, Some examples in fixed point theory ............ 89

Michael Benton Freeman, Tangential Cauchy-Riemann equations and uniform approximation ............................. 101

Barry J. Gardner, Torsion classes and pure subgroups ................ 109

Vinod B. Goyal, Bounds for the solution of a certain class of nonlinear

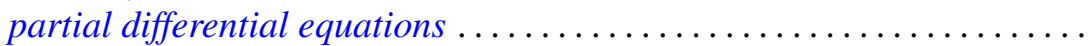

Fu Cheng Hsiang, On C, 1 summability factors of Fourier series at a given

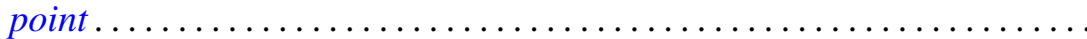

Lawrence Stanislaus Husch, Jr., Homotopy groups of PL-embedding

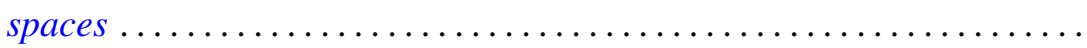

Daniel Ralph Lewis, Integration with respect to vector measures..........

Marion-Josephine Lim, $\mathscr{L}-2$ subspaces of Grassmann product spaces

Stephen J. Pierce, Orthogonal groups of positive definite multilinear functionals

W. J. Pugh and S. M. Shah, On the growth of entire functions of bounded index.

Siddani Bhaskara Rao and Ayyagari Ramachandra Rao, Existence of triconnected graphs with prescribed degrees . . .

Ralph Tyrrell Rockafellar, On the maximal monotonicity of subdifferential

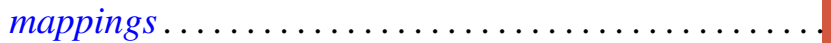

R. Shantaram, Convergence of a sequence of transformations of distribution functions. II ...............................

Julianne Souchek, Rings of analytic functions..............

Ted Joe Suffridge, The principle of subordination applied to functions of several variables...

Wei-lung Ting, On secondary characteristic classes in cobordism

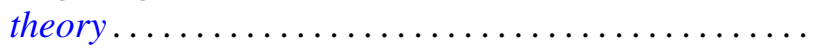

Pak-Ken Wong, Continuous complementors on $B^{*}$-algebras ...

Miyuki Yamada, On a regular semigroup in which the idempotents form a band. 